\title{
Transferência e Transformação de Funções Relacionais de /Maior-Que/ e /Menor-Que/ em Crianças com Deficiência Intelectual
}

\author{
Rosana A. S. Rossit \\ Departamento Saúde, Educação e Sociedade da Universidade Federal de São Paulo, \\ Santos, SP, Brasil \\ Celso Goyos ${ }^{1}$ \\ Departamento de Psicologia da Universidade Federal de São Carlos, São Carlos, SP, Brasil
}

\begin{abstract}
Resumo
O objetivo deste estudo foi verificar a emergência do conceito relacional /menor-que/ e /maior-que/ em crianças com deficiência intelectual, para conjuntos e numerais impressos; e, analisar a emergência de relações derivadas, transformação da função, generalização e produção de respostas. Participaram três crianças com deficiência intelectual, idade entre 8 e 11 anos, matriculadas na rede regular de ensino e experimentalmente ingênuas e com desempenho inferior a $50 \%$ para os conceitos relacionais de /maior-que/ e /menor-que/ no mapeamento do repertório de entrada. Foram utilizados algarismos e conjuntos de 1 a 9, desenhos lineares, símbolos (/igual/, /diferente/,/menor-que/, /maior-que/) e sons gravados correspondentes. O delineamento experimental consistiu de treino de conceito de números e conceitos relacionais; testes imediatos para avaliar as relações emergentes, o responder relacional derivado, transformação da função, generalização, produção de respostas e manutenção após 30 e 60 dias. Os três participantes apresentaram, ao final do estudo, os conceitos numéricos envolvendo os valores de 1 a 9. Também adquiriram o conceito relacional /menor-que/ diretamente ensinado por meio de procedimentos baseados em múltiplos exemplares de tarefas modificadas de MTS, assim como, a transferência do responder relacional deste repertório para relações não diretamente ensinadas e a transferência para novos conceitos relacionais.
\end{abstract}

Palavras-chave: Conceitos matemáticos, conceito relacional, equivalência estímulos, deficiência intelectual.

\section{Transfer and Transformation Functions and Relational/Greater-Than/ and /Lesser-Than/ in Children with Intellectual Disabilities}

\begin{abstract}
The purpose of this study was to investigate the emergence of the concept relational /less-than/ and /greater-than/ in children with intellectual disabilities, for sets and printed numerals; and analyzing

Endereço para correspondência: Departamento de Psicologia, Universidade Federal de São Carlos, Rod. Washington Luís, km 235, São Carlos, SP, Brasil 13565-905. E-mail: rorossit@hotmail.com e celsogoyos2@ gmail.com

O presente artigo teve como base o trabalho de pós-doutorado da primeira autora, sob supervisão do segundo autor. Os autores agradecem ao apoio da Fundação de Amparo à Pesquisa do Estado de São Paulo (FAPESP), através de bolsa de pós-doutorado à primeira autora e através de auxílio à pesquisa (processo 2005/54980-0) ao segundo autor.
\end{abstract}


the emergency of derived relationships, function transformation, generalization and production responses. Participants were three children with intellectual disabilities, aged 8 to 11 years, enrolled in the regular school system and experimentally naive and underperforming the $50 \%$ for relational concepts /greater-than/ and /less-than/ in the register mapping input. Digits were used and sets 1 to 9, line drawings, symbols (/equals/, /other/, /less-than/, /greater-than/ and the corresponding recorded sound. The experimental design consisted of training concept of numbers and relational concepts; Tests to evaluate the immediate emerging relationships derived relational responding, function transformation, generalization, production and maintenance of responses at 30 and 60 days. The three participants presented at the end of the study, the numeric concepts involving the values from 1 to 9 . Also acquired relational concept /less-than/ taught directly by procedures based on multiple copies of modified MTS task, as well as the transfer of relational responding to this repertoire to relations not directly taught and the transference to new relational concepts.

Keywords: Mathematical concepts, relational concepts, stimulus equivalence, intellectual disability.

\section{Transferencia y Transformación de Operadores Relacionales de I Mayor Que/ y /Menor Que/ en Niños con Discapacidad Intelectual}

\section{Resumen}

El objetivo de este estudio fue comprobar la manifestación del concepto relacional /menor que/ y/mayor que/ en niños con discapacidad intelectual, para conjuntos y números impresos; $y$, analizar la manifestación de relaciones derivadas, transformación de la función, generalización y producción de respuestas. Participaron tres niños con discapacidad intelectual, con edades comprendidas entre los 8 y 11 años, matriculadas en la enseñanza ordinaria y experimentalmente ingenuas y con desempeño inferior a $50 \%$ para los conceptos relacionales de /mayor que/y /menor que/ en el levantamiento del repertorio de entrada. Fueron utilizados dígitos y conjuntos de 1 a 9, dibujos lineales, símbolos (/igual/,/diferente/, /menor que/, /mayor que/) y los sonidos gravados correspondientes. El delineamiento experimental consistió en el entrenamiento del concepto de números y conceptos relacionales; pruebas inmediatas para evaluar las relaciones emergentes, la respuesta relacional derivada, transformación de la función, generalización, producción de respuestas y mantenimiento después de 30 y 60 días. Los tres participantes presentaron, al final del estudio, los conceptos numéricos involucrando los valores de 1 a 9. También adquirieron el concepto relacional/menor que/ directamente enseñado por procedimientos basados en múltiples ejemplares de tareas modificadas de MTS, así como, la transferencia de la respuesta relacional de este repertorio para relaciones no directamente enseñadas y la transferencia para nuevos conceptos relacionales.

Palabras clave: Conceptos matemáticos, concepto relacional, equivalencia estímulos, discapacidad intelectual.

Dificuldades na aprendizagem de comportamentos matemáticos são comumente encontradas em estudantes de todas as idades (Neef, Nelles, Iwata, \& Pace, 2003), sendo o equacionamento e a resolução dessas dificuldades importantes para obtenção de sucesso na escola, no trabalho, na independência diária e em situações da vida econômica e social desses indivíduos. A análise do comportamento tem sido aplicada intensamente para investigar e remediar proble- mas derivados das dificuldades na aprendizagem da matemática (para uma revisão, ver Pereira \& Winton, 1991). Dentro desta mesma abordagem, estudos sobre equivalência de estímulos (Sidman, 1971; Stromer, Mackay, \& Stoddard, 1992) lançaram bases promissoras para o ensino de matemática (Green, 1992; Stoddard, Bradley, \& McIlvane, 1987; Stoddard, Brown, Hurlbert, Manoli, \& McIlvane, 1989) com implicações importantes para o ensino de indivíduos com 
atraso no desenvolvimento ou com dificuldades de aprendizagem (ex., De León, 1998; Maydak, Stromer, Mackay, \& Stoddard, 1995; Prado \& de Rose, 1999; Rossit, 2003; Rossit \& Goyos, 2009).

Dentro desta concepção a matemática é vista como uma infinita rede de relações envolvendo estímulos e respostas e essas relações se constituem a base da linguagem matemática.

As relações básicas constituintes dessa linguagem incluem as relações dos operantes verbais ecóico, tato, e textual e o repertório verbal de nomeação (Horne \& Lowe, 1996) de estímulos relativos a dígitos, elementos de conjuntos, conjuntos, a palavras impressas correspondentes, e todas as outras operações, anotações e símbolos matemáticos correspondentes aos dígitos e às relações entre eles e seus símbolos. Os procedimentos de ensino baseados em equivalência de estímulos tornaram-se especialmente atraentes e promissores em função da profusão de relações emergentes que eles produzem, combinada com a análise e organização dos elementos envolvidos na aquisição de comportamentos matemáticos. Isso é conseguido através de uma rede de relações, que possibilitam detectar necessidades individuais de aprendizagem e propiciar a obtenção de informações que subsidiem a elaboração e implementação de estratégias individualizadas de ensino (Stromer et al., 1992).

Apesar dos avanços que as pesquisas sobre equivalência de estímulos produziram no desenvolvimento de tecnologia de ensino de habilidades matemáticas, parte substancial dessa linguagem, no entanto, exige a complementação da análise para além da equivalência dos estímulos (p.ex., Dymond \& Barnes-Holmes, 1995; O'Hora, Roche, Barnes-Holmes, \& Smeets, 2002; Reilly, Whelan, \& Barnes-Holmes, 2005; Steele \& Hayes, 1991; Whelan \& Barnes-Holmes, 2004). As relações diferentes das de igualdade, envolvidas nas operações matemáticas de adição e de subtração, de maior e menor, e diferente ou oposto, são também essenciais a essa linguagem. As propriedades requeridas para que as relações sejam de equivalência: reflexividade, simetria e transitividade, não se aplicam aos desempenhos relacionais envolvidos nas habili- dades, por exemplo, de subtrair ou somar ou da quantidade relativa a essas operações, menos e mais. Essas relações são irreflexivas, assimétricas, transitivas e conectadas (Green, Stromer, \& Mackay, 1993; Ribeiro, Assis, \& Enumo, 2007; Whelan, Barnes-Holmes, \& Dymond, 2006). A simples identificação dessas relações e da diferença delas com as relações presentes em equivalência de estímulos, no entanto, não são suficientes para explicar o fenômeno de emergência de novas relações.

Para explicar essas relações será utilizado o referencial de Dymond e Barnes-Holmes (1995, p. 183). Primeiro, a relação que um estímulo A tem com ele mesmo não é reflexiva: ele não pode ser /mais-que/ ou /menos-que/ ele próprio. Segundo, se A é /mais-que/ $\mathrm{B}$, não procede que $\mathrm{B}$ seja /mais-que/ $A$ : a relação não é simétrica. As relações são transitivas, se $\mathrm{B}$ é /mais-que/ $\mathrm{A}$ e $\mathrm{C}$ é /mais-que/ B, e então C é /mais-que/ $\mathrm{A}$. As relações também são conectadas, isto é, "todo estímulo que participa em uma relação específica /mais-que/ ou /menos-que/ está relacionado um com o outro, e, por conseguinte, a relação é conectada".

Sob a ótica da Teoria dos Quadros Relacionais (RFT) de Hayes, Barnes-Holmes, e Roche, (2001), estudos experimentais têm demonstrado a emergência de responder relacional derivado em uma variedade de situações, muitas delas relevantes à linguagem matemática, incluindo mesmo e oposto (Dymond \& Barnes-Holmes, 1996; Roche, Barnes-Holmes, Smeets, BarnesHolmes, \& McGeady, 2000), diferente (Roche \& Barnes-Holmes, 1996), e/mais-que/ e/menos-que/ (Dymond \& Barnes-Holmes, 1995), dentre outras relações (Ninness et al., 2005).

Na perspectiva da RFT (Hayes et al., 2001), responder de acordo com relações de estímulos inclui as propriedades de derivação mútua, derivação combinatória e a transformação da função².

Com relação à propriedade de derivação mútua, se um dado estímulo é relacionado a outro, tal que a relação /A é o mesmo-que o estí-

2 Do inglês mutual entailment, combinatorial entailment, e transformation of stimulus functions. 
mulo $\mathrm{B} /$, então a relação / $\mathrm{B}$ é o mesmo-que $\mathrm{A} /$, atende à propriedade da derivação mútua $(/ \mathrm{A}=\mathrm{B} /$ então $/ \mathrm{B}=\mathrm{A} /$ ). A propriedade opera em uma maneira consistente com a simetria, dentro da teoria da equivalência de estímulos de Sidman. Por outro lado, se um estímulo A aceita a relação com outro estímulo $\mathrm{B}$, então outra relação derivada entre B e A pode ser estabelecida. Esse tipo de responder relacional depende do tipo de relação treinada diretamente entre A e B. Por exemplo, se o estímulo A aceita a relação /mesmo-que/ $\mathrm{B}$, então a relação / $\mathrm{B}$ é o mesmo-que $\mathrm{A} /$ é aceita. Entretanto, relações treinadas e aceitas nem sempre são idênticas. Nesse caso, dado que /A é mais-que $\mathrm{B} /$, uma relação /menos-que/ é requerida entre B e A, e então, /B é menos-que A/.

Outro tipo de relação pode ocorrer, se /A é o oposto de B/, e /B é o oposto de A/, a mutualidade é requerida. $\mathrm{E}$ se ao mesmo tempo, /B é o oposto de $\mathrm{C} /$, então /C é oposto de B/. Então a partir deste exemplo, a relação /C mesmo que $\mathrm{A} /$ e / $\mathrm{A}$ é o mesmo que $\mathrm{C} /$ é derivada e descrita pela RFT como derivação combinatória. A natureza dessa relação derivada depende da natureza das relações treinadas. Por exemplo, se /A é mais-que B/ e /B é mais-que $\mathrm{C} /$, então uma relação mais-que entre $\mathrm{A}$ e $\mathrm{C}$ é proveniente de uma derivação combinatória - /A é mais-que $\mathrm{C} /$ e /C é menos-que $\mathrm{A} /$.

$\mathrm{Na}$ transformação de função, entretanto, se /A é maior-que B/, então a relação /B é menor-que $\mathrm{A} /$ é requerida.

De forma geral, o presente estudo investigou se o conceito relacional/menor-que/ trei- nado para quantidades e algarismos, poderia ser transferido para quantidades e algarismos não treinados diretamente, e verificar a possibilidade de emergência da transformação da função para /maior-que/.

O estudo teve como objetivos específicos avaliar a emergência dos conceitos relacionais de /maior-que/ e de /menor-que/ em crianças com deficiência intelectual, analisar a generalização dos conceitos para quantidades e algarismos não diretamente ensinados, e analisar as correspondentes transformações de funções.

\section{Método}

\section{Participantes}

Participaram desta pesquisa três crianças com deficiência intelectual, com idade entre oito e 11 anos, ambos os gêneros, matriculadas na rede regular de ensino e sem experiência anterior com procedimentos de discriminação condicional em tarefas de escolha de acordo com o modelo computadorizadas. No mapeamento do repertório matemático para valores de um a três, os participantes Jov e Jul apresentaram desempenhos inferiores para a relação AB (algarismo ditado-quantidades) e apresentaram as relações de tato relativas a quantidades e algarismos (relação $\mathrm{BD}$ e $\mathrm{CD}$ ) de forma inconsistente. Rod apresentou o conceito de números para valores de um a três. Nenhum dos participantes apresentou desempenho superior a $50 \%$ para os conceitos relacionais de maior-que e menor-que. A Tabela 1 fornece a caracterização dos participantes.

\section{Tabela 1}

\section{Caracterização dos Participantes}

\begin{tabular}{cccccc}
\hline Participantes & $\begin{array}{c}\text { Idade } \\
(*)\end{array}$ & Gênero & $\begin{array}{c}\text { Diagnóstico } \\
(* *)\end{array}$ & $\begin{array}{c}\text { Velocidade de } \\
\text { processamento }(* *)\end{array}$ & $\begin{array}{c}\text { Escolarização } \\
(* * *)\end{array}$ \\
\hline Jov & 10,9 & $\mathrm{M}$ & Intelectualmente deficiente & Média & $3^{\mathrm{a}}$ série \\
Jul & 8,5 & $\mathrm{~F}$ & Limítrofe & Média & $1^{\mathrm{a}}$ série \\
Rod & 12 & $\mathrm{M}$ & Intelectualmente deficiente & Média & $4^{\mathrm{a}}$ série \\
\hline
\end{tabular}

Notas. * Anos, meses, no início do estudo. ** Escala Wechsler de Inteligência para Crianças aplicado em Setembro de 2005 (Wechsler, 1991). *** Escola regular. 


\section{Local, Ambiente Experimental e Materiais}

$\mathrm{O}$ ambiente experimental foi montado em uma sala, medindo aproximadamente $9,0 \mathrm{~m}^{2}$ contendo uma mesa, duas cadeiras e um microcomputador $i M a c$ multimídia com monitor colorido, com programa computacional Mestre $^{\circledR}$ (Goyos \& Almeida, 1994) instalado, utilizado para programar, conduzir, registrar e arquivar os dados. Foram também utilizadas fichas plásticas, um tubo de P.V.C. para liberação de fichas e um recipiente transparente para armazenamento de fichas.

\section{Estímulos Experimentais}

Foram utilizados como estímulos: quantidades em conjunto de 1 a 9 bolinhas, os algarismos de 1 a 9 na cor preta, tamanho 72 e três desenhos lineares em cor preta (óculos, tesoura e telefone). Foram também utilizados estímulos visuais (símbolo de /igual/, /diferente/, /menor-que/ e / maior-que/) e os respectivos sons, gravados previamente em arquivos digitais. Todos os estímulos foram apresentados em uma janela branca medindo $4,0 \mathrm{~cm}$ por $4,0 \mathrm{~cm}$.

\section{Procedimentos}

Levantamento de Itens de Preferência. Nesse procedimento, descrito detalhadamente em Escobal e Goyos (2008), foram utilizados dez objetos identificados a partir do relato verbal dos participantes os quais mapearam os interesses individuais. Os itens escolhidos foram hierarquizados e recombinados de acordo com a preferência e reapresentados. As preferências finais foram definidas e classificadas: Nível Alto (NA) - três ou mais escolhas; Nível Médio (NM) duas escolhas; Nível Baixo (NB) - uma escolha; e Sem Preferência (SP) - nenhuma escolha. Três itens, correspondentes aos três níveis de menor preferência foram inicialmente disponibilizados para que o participante pudesse trocar suas fichas após terem alcançado critério de $90 \%$ de desempenho correto. Os itens com NA de preferência só foram disponibilizados ao final de cada estudo.
Familiarização com o Sistema Computacional. O ensino dos componentes comportamentais da tarefa de escolha de acordo com o modelo (matching-to-sample) foi introduzido através de três relações de identidade. Uma tentativa da tarefa era iniciada com a apresentação de um estímulo modelo visual. A resposta de observação, definida como um toque sobre o estímulo, foi seguida pela apresentação de três estímulos de comparação correspondentes a figuras de objetos. Escolhas do estímulo idêntico ao estímulo modelo eram seguidas pela entrega de uma ficha, que era depositada em um recipiente transparente. Escolhas do estímulo não idêntico ao estímulo modelo eram seguidas pela remoção de todos os estímulos da tela, apresentação de um intervalo inter-tentativas de 2 s, e apresentação de uma nova tentativa. Ao final da sessão com critério de $90 \%$ de escolhas corretas, as fichas acumuladas eram trocadas por um item de preferência e o participante avançava para a fase seguinte. Abaixo desse índice, as fichas eram recolhidas e uma nova sessão era iniciada. As tentativas foram agrupadas em um bloco com 18 unidades, sendo que cada uma das três possíveis relações de identidade era apresentada em igual número. Para cada relação, a posição dos estímulos corretos era balanceada e este era apresentado na mesma posição por, no máximo, duas tentativas consecutivas.

\section{Delineamento Experimental}

A Tabela 2 mostra a ordem pela qual as etapas experimentais foram apresentadas, as relações ensinadas e testadas, a natureza dos conceitos - numérico ou relacional - seus respectivos valores e número de tentativas utilizadas por sessão. Inicialmente um pré-teste foi aplicado para os valores 1,2 , e 3 , envolvendo as relações condicionais para o conceito numérico e as relações para os conceitos/menor-que/ e /maior-que/, para os mesmos valores. Em seguida, introduziu-se o ensino das relações identificadas com desempenho inferior a $90 \%$ de escolhas corretas, iniciando-se pelo ensino das relações $\mathrm{AB}$ e $\mathrm{BC}$, e dos testes para verificação da emergência das relações $\mathrm{BC}, \mathrm{CB}, \mathrm{BD}$ e $\mathrm{CD}$. Após esses testes, 
foi introduzido o ensino do conceito relacional /menor-que/ envolvendo as operações $/ 1<2 / \mathrm{e}$ $/ 2<3 /$, seguido dos testes da emergência do mesmo conceito relacional envolvendo a operação $/ 1<3 /$ e dos testes para verificação da transformação da função /menor-que/ para /maior-que/, envolvendo as operações $/ 2>1 /, / 3>2 /, / 3>1 /$, e, também, dos testes de generalização para todas as relações envolvendo os valores 4, 5, 6. A fase que se seguiu foi análoga à anterior, substituindo-se os valores $1,2,3$ por $4,5,6$ e os valores $4,5,6$, por $7,8,9$. A última fase consistiu do ensino es dos testes do conceito numérico e do conceito relacional para os valores $7,8,9$.

Tabela 2

Ordem pela qual as Etapas Experimentais foram Apresentadas, Natureza das Relações - Ensino e Teste, Valores e Estímulos para o Conceito Numérico e Conceito Relacional e o Número Total de Tentativas

\begin{tabular}{|c|c|c|c|c|c|}
\hline \multirow{2}{*}{ Item } & \multicolumn{2}{|r|}{ Natureza } & \multicolumn{2}{|c|}{ Valores/Estímulos } & \multirow{2}{*}{$\begin{array}{l}\text { № Total de } \\
\text { Tentativas }\end{array}$} \\
\hline & ENSINO/TESTES & RELAÇÕES & $\begin{array}{c}\text { Conceito } \\
\text { Numérico }\end{array}$ & $\begin{array}{l}\text { Conceito } \\
\text { Relacional }\end{array}$ & \\
\hline \multirow{3}{*}{1} & \multirow{3}{*}{ PRÉ-TESTE GERAL } & $\mathrm{AB}, \mathrm{AC}, \mathrm{BC}, \mathrm{CB}, \mathrm{DB}, \mathrm{DC}$ & $1,2,3$ & & 54 \\
\hline & & $\mathrm{B}<\mathrm{B}, \mathrm{B}<\mathrm{C}, \mathrm{C}<\mathrm{C}, \mathrm{C}<\mathrm{B}$ & & $1<2,1<3,2<3$ & 12 \\
\hline & & $\mathrm{B}>\mathrm{B}, \mathrm{B}>\mathrm{C}, \mathrm{C}>\mathrm{C}, \mathrm{C}>\mathrm{B}$ & & $2>1,3>2,3>1$ & 12 \\
\hline 2 & ENSINO & $\mathrm{AB}$ e $\mathrm{AC}$ & $1,2,3$ & & 18 \\
\hline \multirow{2}{*}{3} & TESTE & $\mathrm{BC}, \mathrm{CB}$ & $1,2,3$ & & 18 \\
\hline & TESTE & DB, DC & $1,2,3$ & & 18 \\
\hline 4 & ENSINO & $\mathrm{B}<\mathrm{B}, \mathrm{B}<\mathrm{C}, \mathrm{C}<\mathrm{C}, \mathrm{C}<\mathrm{B}$ & & $1<2$ e $2<3$ & 24 \\
\hline \multirow{3}{*}{5} & \multirow{3}{*}{$\begin{array}{c}\text { TESTE } \\
\text { Generalização }\end{array}$} & $\mathrm{B}<\mathrm{B}, \mathrm{B}<\mathrm{C}, \mathrm{C}<\mathrm{C}, \mathrm{C}<\mathrm{B}$ & & $1<3$ & 12 \\
\hline & & $\mathrm{B}>\mathrm{B}, \mathrm{B}>\mathrm{C}, \mathrm{C}>\mathrm{C}, \mathrm{C}>\mathrm{B}$ & & $2>1,3>2,3>1$ & 24 \\
\hline & & $\begin{array}{c}\mathrm{AB}, \mathrm{AC}, \mathrm{BC}, \mathrm{CB}, \mathrm{DB}, \mathrm{DC} \\
\mathrm{B}<\mathrm{B}, \mathrm{B}<\mathrm{C}, \mathrm{C}<\mathrm{C}, \mathrm{C}<\mathrm{B}\end{array}$ & $4,5,6$ & $4<5$ e $5<6$ & $\begin{array}{l}54 \\
24\end{array}$ \\
\hline 6 & ENSINO & $\mathrm{AB}$ e $\mathrm{AC}$ & $4,5,6$ & & 18 \\
\hline \multirow{2}{*}{7} & TESTE & $\mathrm{BC}, \mathrm{CB}$ & $4,5,6$ & & 18 \\
\hline & TESTE & $\mathrm{DB}, \mathrm{DC}$ & $4,5,6$ & & 18 \\
\hline 8 & ENSINO & $\mathrm{B}<\mathrm{B}, \mathrm{B}<\mathrm{C}, \mathrm{C}<\mathrm{C}, \mathrm{C}<\mathrm{B}$ & & $4<5$ e $5<6$ & 24 \\
\hline \multirow{3}{*}{9} & \multirow{2}{*}{ TESTE } & $\mathrm{B}<\mathrm{B}, \mathrm{B}<\mathrm{C}, \mathrm{C}<\mathrm{C}, \mathrm{C}<\mathrm{B}$ & & $4<6$ & 12 \\
\hline & & $\mathrm{B}>\mathrm{B}, \mathrm{B}>\mathrm{C}, \mathrm{C}>\mathrm{C}, \mathrm{C}>\mathrm{B}$ & & $5>4,6>5,6>4$ & 24 \\
\hline & Generalização & $\begin{array}{c}\mathrm{AB}, \mathrm{AC}, \mathrm{BC}, \mathrm{CB}, \mathrm{DB}, \mathrm{DC} \\
\mathrm{B}<\mathrm{B}, \mathrm{B}<\mathrm{C}, \mathrm{C}<\mathrm{C}, \mathrm{C}<\mathrm{B}\end{array}$ & $7,8,9$ & $7<8$ e $8<9$ & $\begin{array}{l}54 \\
24\end{array}$ \\
\hline 10 & ENSINO & $\mathrm{AB}$ e $\mathrm{AC}$ & $7,8,9$ & & 18 \\
\hline \multirow{2}{*}{11} & TESTE & $\mathrm{BC}, \mathrm{CB}$ & $7,8,9$ & & 18 \\
\hline & TESTE & $\mathrm{DB}, \mathrm{DC}$ & $7,8,9$ & & 18 \\
\hline 12 & ENSINO & $\mathrm{B}<\mathrm{B}, \mathrm{B}<\mathrm{C}, \mathrm{C}<\mathrm{C}, \mathrm{C}<\mathrm{B}$ & & $7<8$ e $8<9$ & 24 \\
\hline \multirow{2}{*}{13} & \multirow{2}{*}{ TESTE } & $\mathrm{B}<\mathrm{B}, \mathrm{B}<\mathrm{C}, \mathrm{C}<\mathrm{C}, \mathrm{C}<\mathrm{B}$ & & $7<9$ & 12 \\
\hline & & $\mathrm{B}>\mathrm{B}, \mathrm{B}>\mathrm{C}, \mathrm{C}>\mathrm{C}, \mathrm{C}>\mathrm{B}$ & & $8>7,9>8,9>7$ & 24 \\
\hline
\end{tabular}




\section{Pré-Teste Geral}

Conceito Numérico. Foram testadas individualmente as relações $\mathrm{AB}$ (palavra ditada quantidade), AC (palavra ditada - algarismo), $\mathrm{BC}$ (quantidade - algarismo), CB (algarismo quantidade), $\mathrm{BD}$ (tato da quantidade) e CD (tato do algarismo). O diagrama da Figura 1 ilustra as relações referentes ao conceito de número. As tarefas de teste das relações $\mathrm{AB}, \mathrm{AC}, \mathrm{BC}$ e $\mathrm{CB}$ foram semelhantes às do procedimento para a familiarização com a tarefa de escolha de acordo com o modelo. No entanto, essas tarefas eram de pareamento arbitrário. Adicionalmente, nas relações $\mathrm{AB}$ e $\mathrm{AC}$, os estímulos modelos eram auditivos. Em todas as relações pré-testadas as escolhas foram seguidas somente pelo intervalo inter-tentativas. No teste das relações BD e CD uma tentativa era iniciada, respectivamente, com a apresentação do estímulo quantidade, seguido da instrução: "quantas têm?"; e do estímulo algarismo seguido da instrução "que número é esse?". Respostas orais, independentemente de serem corretas ou incorretas, eram seguidas por um intervalo inter-tentativas de $2 \mathrm{~s}$ e pela apresentação da tentativa seguinte. Os testes para cada relação eram compostos por 54 tentativas, sendo 9 tentativas para cada relação, envolvendo os valores 1,2 , e 3 , e apresentadas em blocos separadamente por tipo de relação.

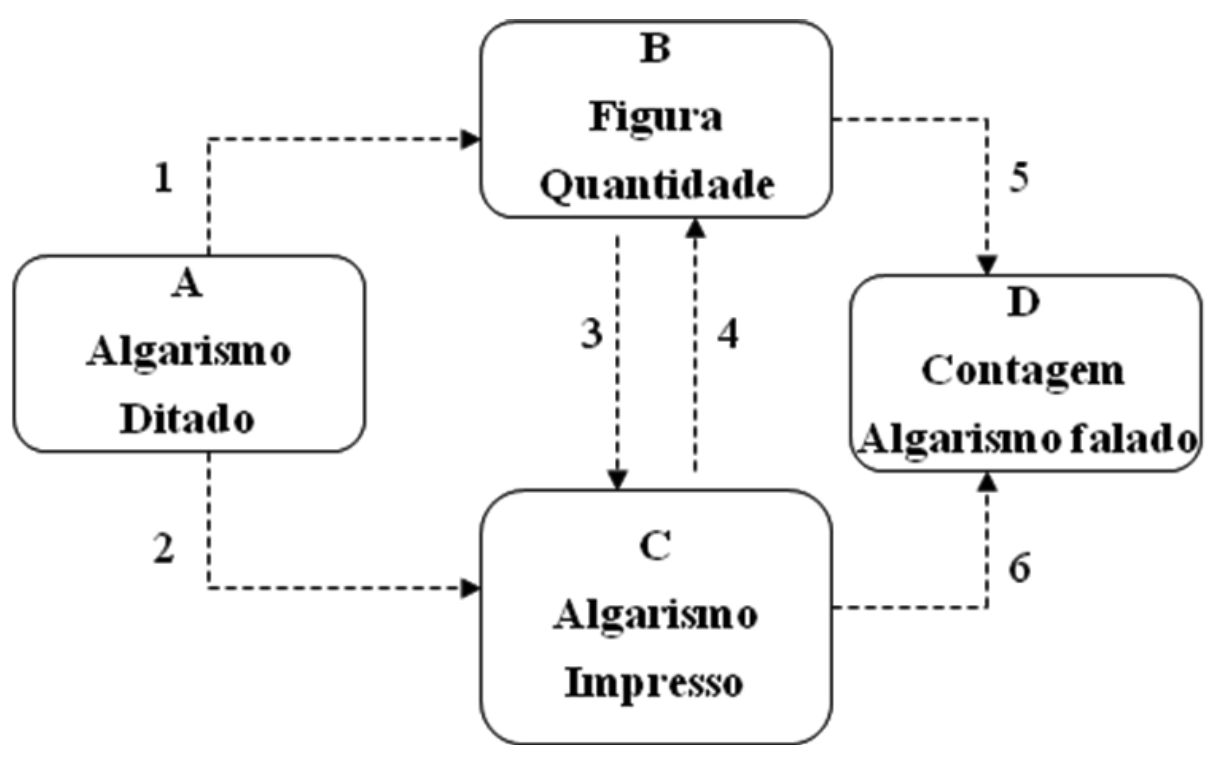

Figura 1. Diagrama esquemático das relações testadas para definir o repertório de entrada. "A" corresponde ao algarismo ditado; " $\mathrm{B}$ " corresponde a quantidades; "C" corresponde ao algarismo; e " $D$ " corresponde à nomeação da quantidade $(B)$ e do algarismo impresso $(C)$. As linhas tracejadas representam as relações testadas.

Conceito Relacional de /Menor-Que/ e de / Maior-Que/. Uma tentativa de teste era iniciada com a apresentação do estímulo modelo do conjunto B (quantidade-bolinhas) ou do conjunto $\mathrm{C}$ (algarismo impresso). Após a resposta de observação, apresentava-se a instrução oral, /menor-que/ ou /maior-que/, e após 2s apresentavam-se três estímulos de comparação, dois deles selecionados dentre os elementos dos conjuntos $\mathrm{B}$ e C, e um terceiro estímulo distrator (Ver Figuras 2 e 3). As respostas de escolha, independentemente de serem corretas ou incorretas, eram se- guidas por um intervalo inter-tentativas de $2 \mathrm{~s}$ e pela apresentação da próxima tentativa.

As tentativas foram apresentadas combinando-se, de maneira semi-randômica os estímulos $\mathrm{B}$ e $\mathrm{C}$ para os valores 1,2 , e 3 . As relações $/ \mathrm{B}_{1,2}$ menor-que $\mathrm{B}_{2,3} / ; / \mathrm{B}_{1,2}$ menor-que $\mathrm{C}_{2,3} / ; / \mathrm{C}_{1,2}$ menor-que $\mathrm{C}_{2,3} / \mathrm{e} / \mathrm{C}_{1,2}$ menor-que $\mathrm{B}_{2,3} /$, combinadas com os valores $/ 1<2 / ; / 1<3 /$ e $/ 2<3 /$, totalizaram 12 tentativas. As relações $/ \mathrm{B}_{2,3}$ maior-que $\mathrm{B}_{1,2} / ; /$ $\mathrm{B}_{2,3}$ maior-que $\mathrm{C}_{1,2} / ; / \mathrm{C}_{2,3}$ maior-que $\mathrm{C}_{1,2} /$ e $/ \mathrm{C}_{2,3}$ maior-que $\mathrm{B}_{1,2} /$ combinadas com os valores $/ 3>2 /$; $/ 3>1 /$ e $/ 2>1 /$, também totalizaram 12 tentativas. 


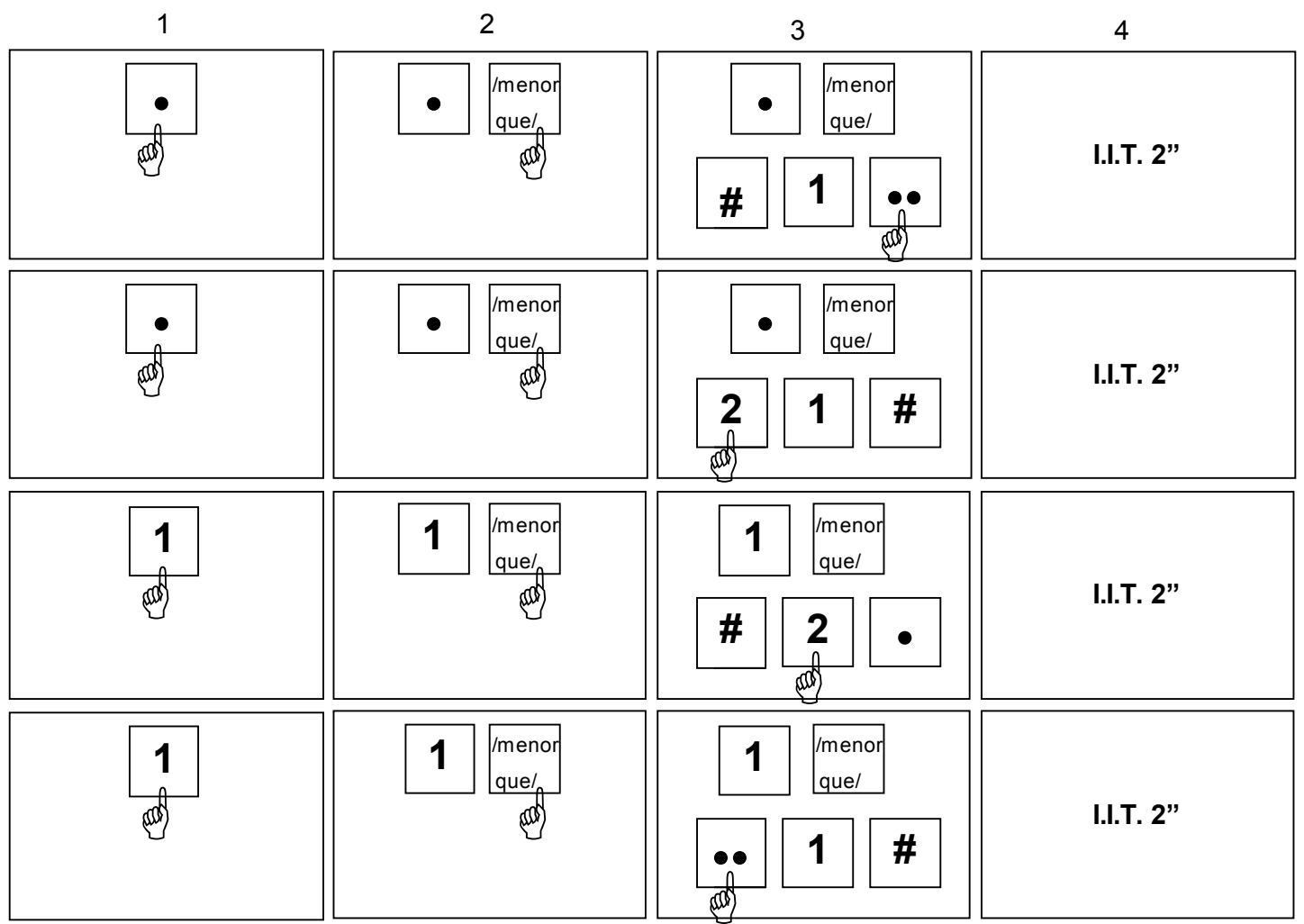

Figura 2. Sequência das telas do computador com as possibilidades de arranjo das tentativas para o conceito relacional de $/ 1<2 /$.

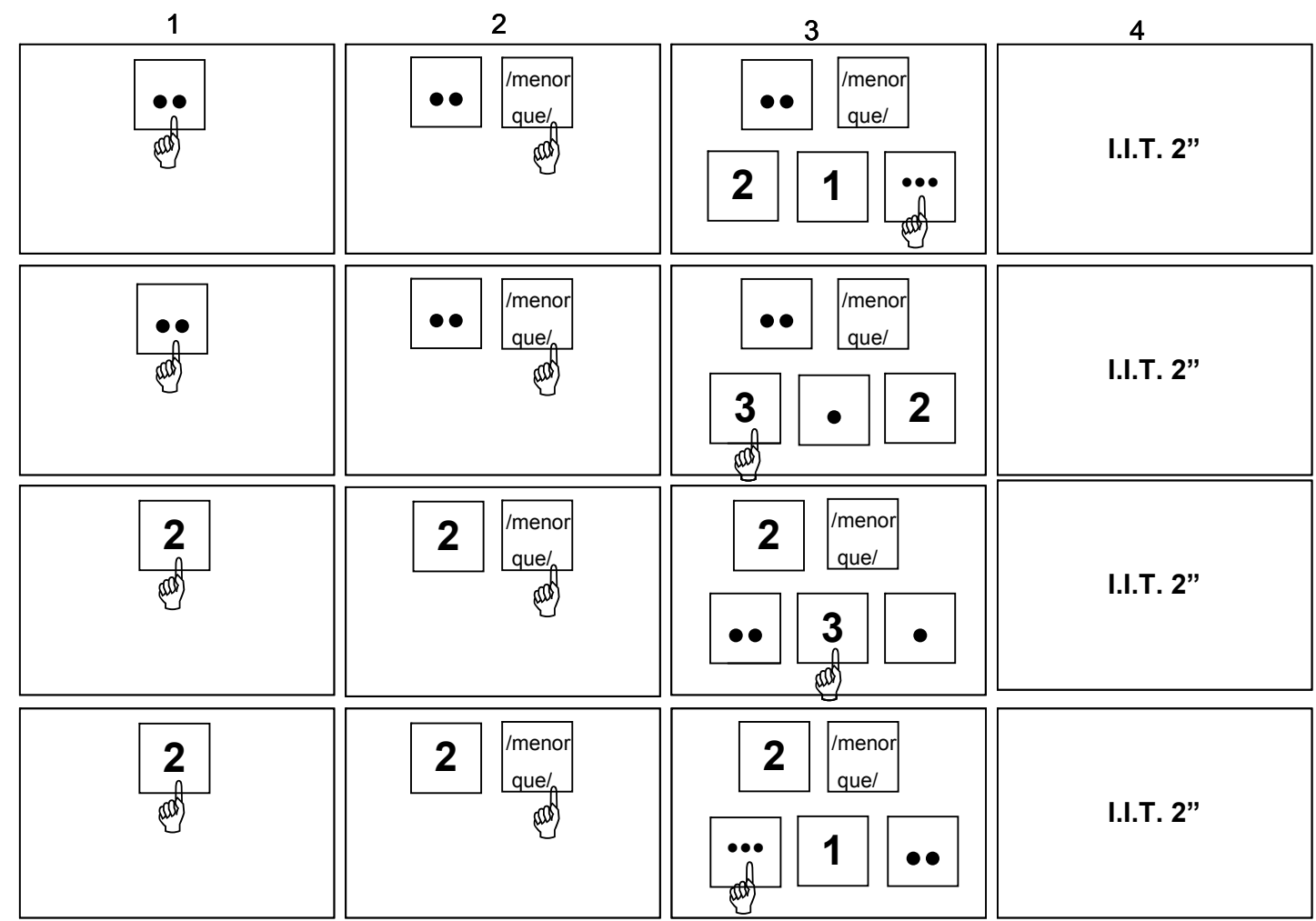

Figura 3. Sequência das telas do computador com as possibilidades de arranjo das tentativas para o conceito relacional de $/ 2<3 /$. 


\section{Procedimento de Ensino}

Conceitos Numéricos. As relações $\mathrm{AB}$ e $\mathrm{AC}$ foram ensinadas separadamente, através da tarefa de escolha de acordo com o modelo, em sessões constituídas por 9 tentativas para cada relação, envolvendo os valores $1,2,3$. A apresentação das tentativas seguiu o mesmo procedimento descrito para a familiarização com a tarefa. Consequências diferenciais foram utilizadas: escolhas consistentes eram seguidas de uma ficha e um elogio verbal; e, escolhas inconsistentes eram seguidas do intervalo inter-tentativas. O critério de aprendizagem de cada relação e da mudança de fase foi de $90 \%$ de escolhas consistentes em uma sessão. Após os testes de relações emergentes e de generalização para os valores 1,2 , e 3 foram inseridos os valores 4,5 , e 6 ; e depois os valores 7,8 , e 9 com procedimento análogo.

Conceito Relacional. Nas tentativas envolvendo o ensino do conceito relacional apresentava-se o algarismo ou quantidade como estímulo modelo. Após a resposta de observação apresentava-se o estímulo auditivo relacional / menor-que/ ou /maior-que/. Na sequência eram apresentados os três estímulos de comparação. As conseqüências para escolhas consistentes e inconsistentes foram análogas ao do ensino do conceito de número. Iniciou-se o ensino com os valores 1 , 2, e 3, seguido dos testes de relações emergentes e de generalização. Os valores 4,5 , e 6, e depois, 7, 8, e 9 foram inseridos com procedimento análogo. O critério de aprendizagem e de mudança de fase foi o mesmo $(90 \%)$.

\section{Procedimento de Testes}

Os testes de cada tipo de relação sempre foram aplicados em uma única sessão, com as tentativas distribuídas de maneira semi-randômica entre as tentativas de linha de base.

Conceitos Numéricos. As sessões de teste das relações $\mathrm{BC}$ e $\mathrm{CB}$ foram constituídas por 18 tentativas, distribuídas de maneira semi-randômica entre as tentativas de linha de base $\mathrm{AB}$ e AC. A forma de apresentação foi similar à do ensino, exceto que a única consequência programada foi a apresentação do intervalo de $2 \mathrm{~s}$.
As relações $\mathrm{BD}$ e $\mathrm{CD}$ foram testadas através da relação de tato (CD) ou contagem (BD), constituídas por 18 tentativas envolvendo os valores 1, 2, e 3. Nas tarefas envolvendo as relações de contagem e de tato, o procedimento de coleta de dados era realizado manualmente pelo experimentador, através de acionar o número " 1 " do teclado numérico para a contagem/tato consistente ou acionar o número " 3 " para a resposta de contagem/tato inconsistente. Um observador independente registrou as respostas em protocolo contendo a sequência dos conjuntos ou algarismos e as respostas para cada tentativa.

Conceito Relacional. A linha de base foi formada pela combinação das relações $/ \mathrm{B}<\mathrm{B} /$; / $\mathrm{B}^{3}<\mathrm{C}^{4} / ; / \mathrm{C}<\mathrm{C} /$ e $/ \mathrm{C}<\mathrm{B} /$, com os valores $/ 1<2 /$ e $/ 2<3 /$. A relação $/ 1<3 /$ foi testada em sessão única através de tarefa de escolha de acordo com o modelo envolvendo conceito relacional, constituída de 12 tentativas. Quatro tentativas para a relações $1<3$ foram distribuídas de maneira semi-randômica entre as tentativas de linha de base. As escolhas eram seguidas apenas do intervalo inter-tentativas. As relações $/ 3>2 /, / 3>1 /$ e $/ 2>1 /$ foram testadas através da combinação das relações $/ \mathrm{B}>\mathrm{B} /$; $/ \mathrm{B}>\mathrm{C} /$; $/ \mathrm{C}>\mathrm{C} / \mathrm{e} / \mathrm{C}>\mathrm{B} /$, em uma única sessão através de tarefa de escolha de acordo com o modelo envolvendo conceito relacional, constituída de 24 tentativas. Três tentativas para cada uma das relações $/ 3>2 /, / 3>1 / \mathrm{e}$ $/ 2>1 /$ foram distribuídas de maneira semi-randômica entre as tentativas de linha de base.

Generalização. Após a aplicação dos testes, e obtenção do critério de aprendizagem com os valores 1,2 , e 3 , para o conceito numérico $(\mathrm{AB}$, $\mathrm{AC}, \mathrm{BC}, \mathrm{CB}, \mathrm{DB}, \mathrm{DC})$ e para o conceito relacional ( $/ \mathrm{B}<\mathrm{B} /, / \mathrm{B}<\mathrm{C} /, / \mathrm{C}<\mathrm{C} /, / \mathrm{C}<\mathrm{B} /$ ) aplicou-se o teste de generalização dessas mesmas relações para os valores 4,5 , e 6 , seguindo-se procedimento análogo ao descrito para os valores 1,2 , e 3. Procedeu-se da mesma maneira para inserir o ensino e testes dos valores 7,8 , e 9 .

\footnotetext{
3 Conjunto $\mathrm{B}=$ quantidade bolinha.

4 Conjunto $\mathrm{C}=$ número impresso.
} 


\section{Resultados}

\section{Pré-Teste Geral}

A Tabela 3 mostra o desempenho de Jov, Jul e Rod nos pré-testes das relações de conceito numérico, $\mathrm{AB}, \mathrm{AC}, \mathrm{BD}, \mathrm{CD}, \mathrm{BC}$ e $\mathrm{CB}$, e a Tabela 4 , o de conceito relacional $/ 1<2 /, / 1<3 /, / 2<3 /$, $/ 2>1 /, / 3>2 /$ e $/ 3>1 /$.
Com relação ao conceito numérico, Rod exibiu desempenho de $100 \%$ de acertos em todas as relações, e Jov e Jul apresentaram desempenho fora do critério em grande parte das relações envolvendo os algarismos 2 e 3 , os estímulos de quantidades, e também nas tarefas de contagem. Com relação aos conceitos relacionais, todos os participantes apresentaram desempenho fora do critério.

Tabela 3

Porcentagem de Acertos no Pré-Teste para as Relações do Conceito Numérico para os Valores 1, 2 e 3

\begin{tabular}{|c|c|c|c|c|c|c|c|c|c|c|c|c|c|c|c|c|c|c|}
\hline & \multicolumn{18}{|c|}{ Relações Conceito Numérico/Valores } \\
\hline & \multicolumn{3}{|c|}{$\mathrm{AB}$} & \multicolumn{3}{|c|}{$\mathrm{AC}$} & \multicolumn{3}{|c|}{$\mathrm{BD}$} & \multicolumn{3}{|c|}{$\mathrm{CD}$} & \multicolumn{3}{|c|}{$\mathrm{BC}$} & \multicolumn{3}{|c|}{$\mathrm{CB}$} \\
\hline & 1 & 2 & 3 & 1 & 2 & 3 & 1 & 2 & 3 & 1 & 2 & 3 & 1 & 2 & 3 & 1 & 2 & 3 \\
\hline Jov & 100 & 66 & 33 & 100 & 100 & 100 & 66 & 33 & 33 & 100 & 100 & 100 & 100 & 66 & 33 & 100 & 100 & 66 \\
\hline Jul & 100 & 100 & 66 & 100 & 100 & 66 & 100 & 100 & 33 & 100 & 100 & 100 & 100 & 66 & 66 & 100 & 100 & 66 \\
\hline Rod & 100 & 100 & 100 & 100 & 100 & 100 & 100 & 100 & 100 & 100 & 100 & 100 & 100 & 100 & 100 & 100 & 100 & 100 \\
\hline
\end{tabular}

Tabela 4

Porcentagem de Acertos no Pré-Teste para as Relações do Conceito Relacional para os Valores 1, 2 e 3

\begin{tabular}{|c|c|c|c|c|c|c|}
\hline & \multicolumn{6}{|c|}{ Relações Conceito Relacional/Valores } \\
\hline & $1<2$ & $2<3$ & $2>1$ & $3>2$ & $3>1$ & $1<3$ \\
\hline Jov & 50 & 50 & 50 & 75 & 25 & 25 \\
\hline Jul & 50 & 70 & 33,3 & 33,3 & 33,3 & 33,3 \\
\hline Rod & 0 & 0 & 0 & 0 & 0 & 0 \\
\hline
\end{tabular}

\section{Ensino e Testes}

As Figuras 4, 5 e 6 apresentam os resultados individuais para Jov, Jul e Rod, respectivamente, referentes ao ensino e aos testes das relações dos conceitos numéricos e relacionais. Os pai- néis superiores, intermediários, e inferiores das figuras referem-se, respectivamente, aos desempenhos nos valores 1,2 , e 3; 4, 5, e 6; e 7, 8, e 9 .

Os resultados de Jov e Jul serão apresentados conjuntamente, em função da semelhança observada entre seus desempenhos. 

em Crianças com Deficiência Intelectual.

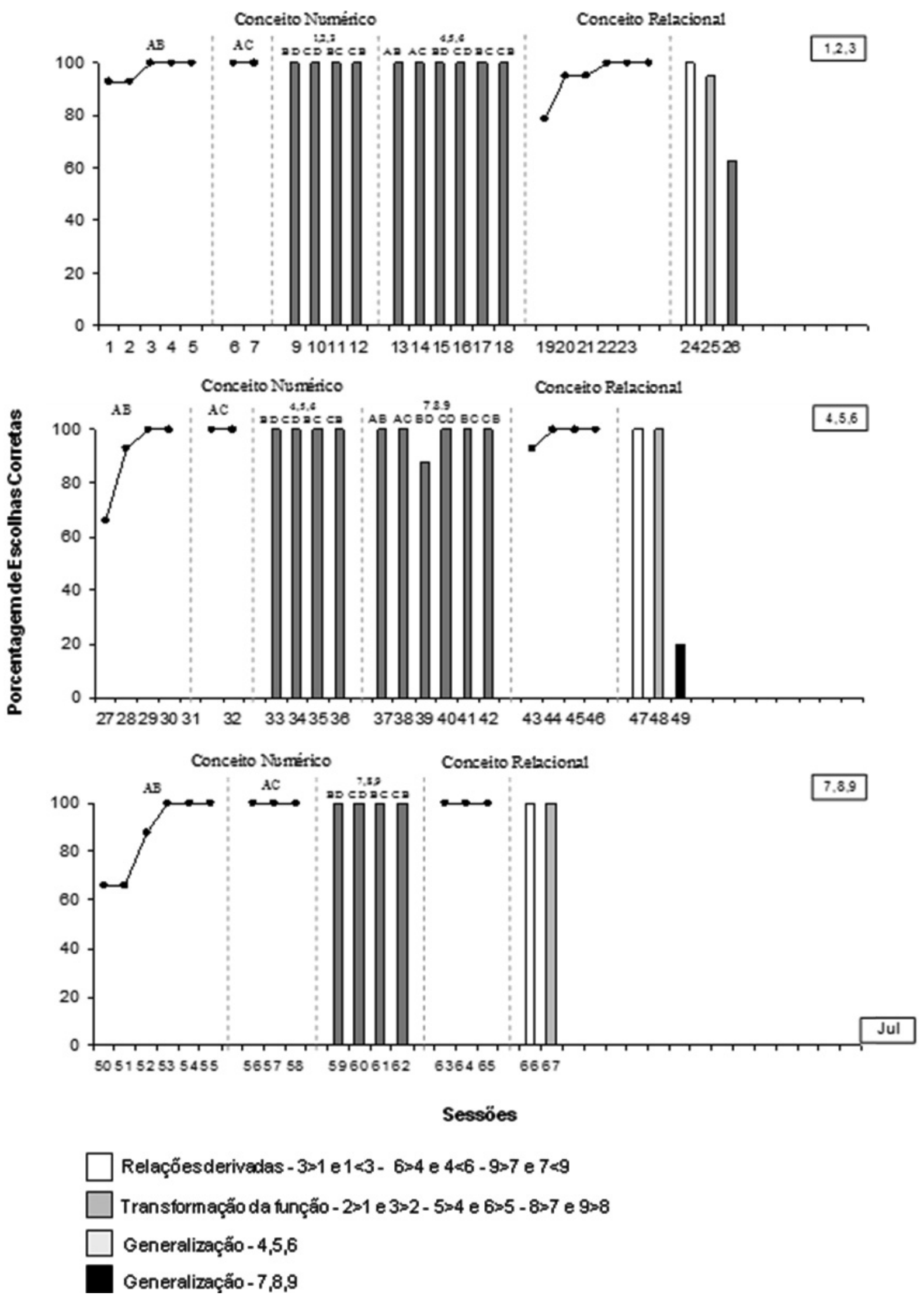

Figura 4. Desempenhos de Jul no ensino e testes das relações para os conceitos numéricos e conceitos relacionais para os valores um a nove. Os pontos ligados por linhas ilustram as relações ensinadas diretamente e as barras ilustram as relações testadas. Os painéis superior, intermediário e inferior referem-se, respectivamente, aos resultados com os valores 1, 2, 3; 4, 5, 6 e 7, 8, 9 , exceto quando especificado pela legenda. 

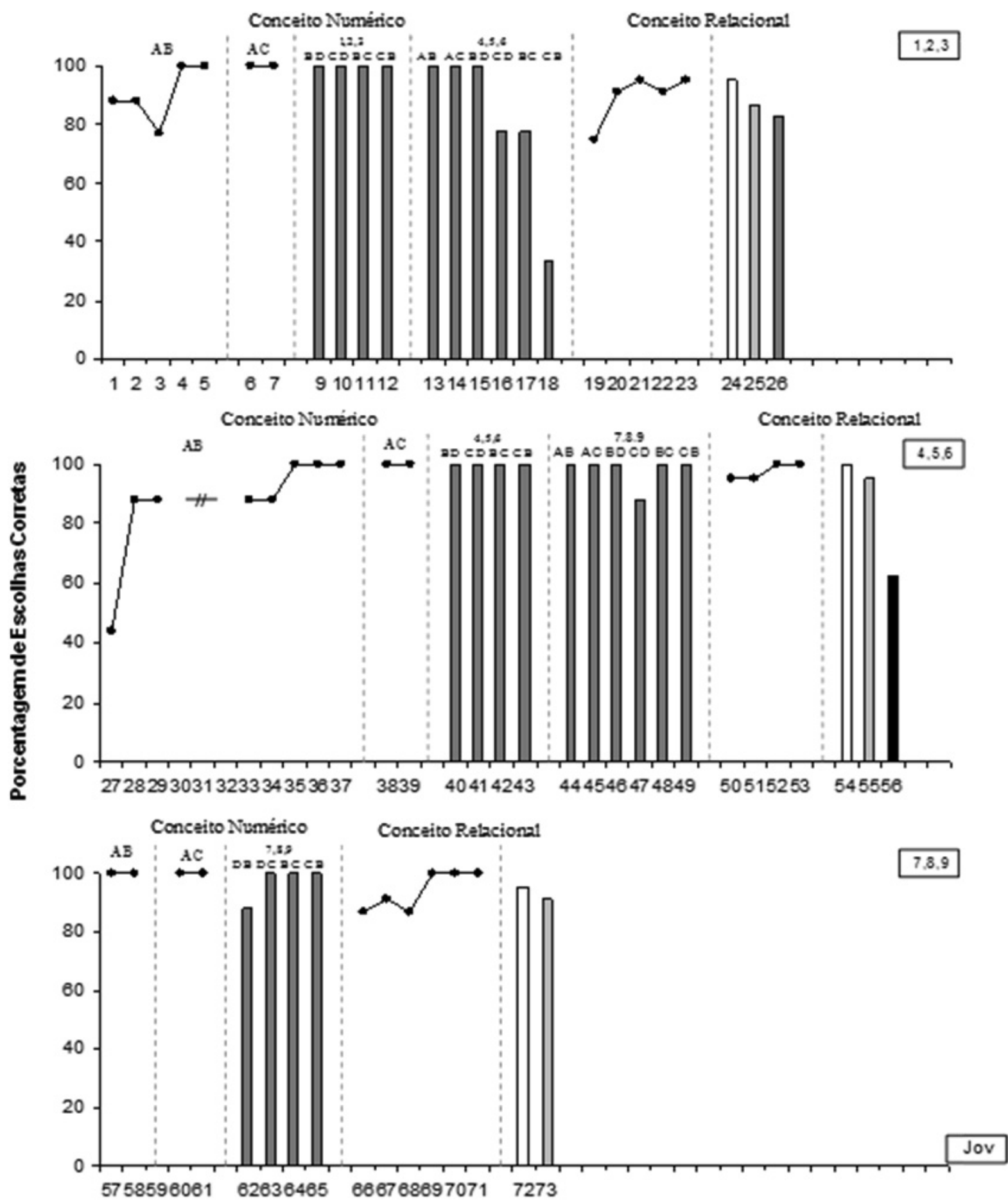

Sessöes

Relaçốes derivadas - $3>1$ e $1<3-6>4$ e $4<6$ - $9>7$ e $7<9$

Transformação da funçẫo - $2>1$ e $3>2-5>4$ e $6>5-8>7$ e $9>8$

Generalizaçăo $-4,5,6$

Generalização - 7,8,9

Figura 5. Desempenhos de Jov no ensino e testes das relações para os conceitos numéricos e conceitos relacionais para os valores um a nove. Os pontos ligados por linhas ilustram as relações ensinadas diretamente e as barras ilustram as relações testadas. Os painéis superior, intermediário e inferior referem-se, respectivamente, aos resultados com os valores $1,2,3 ; 4,5$, 6 e $7,8,9$, exceto quando especificado pela legenda. 

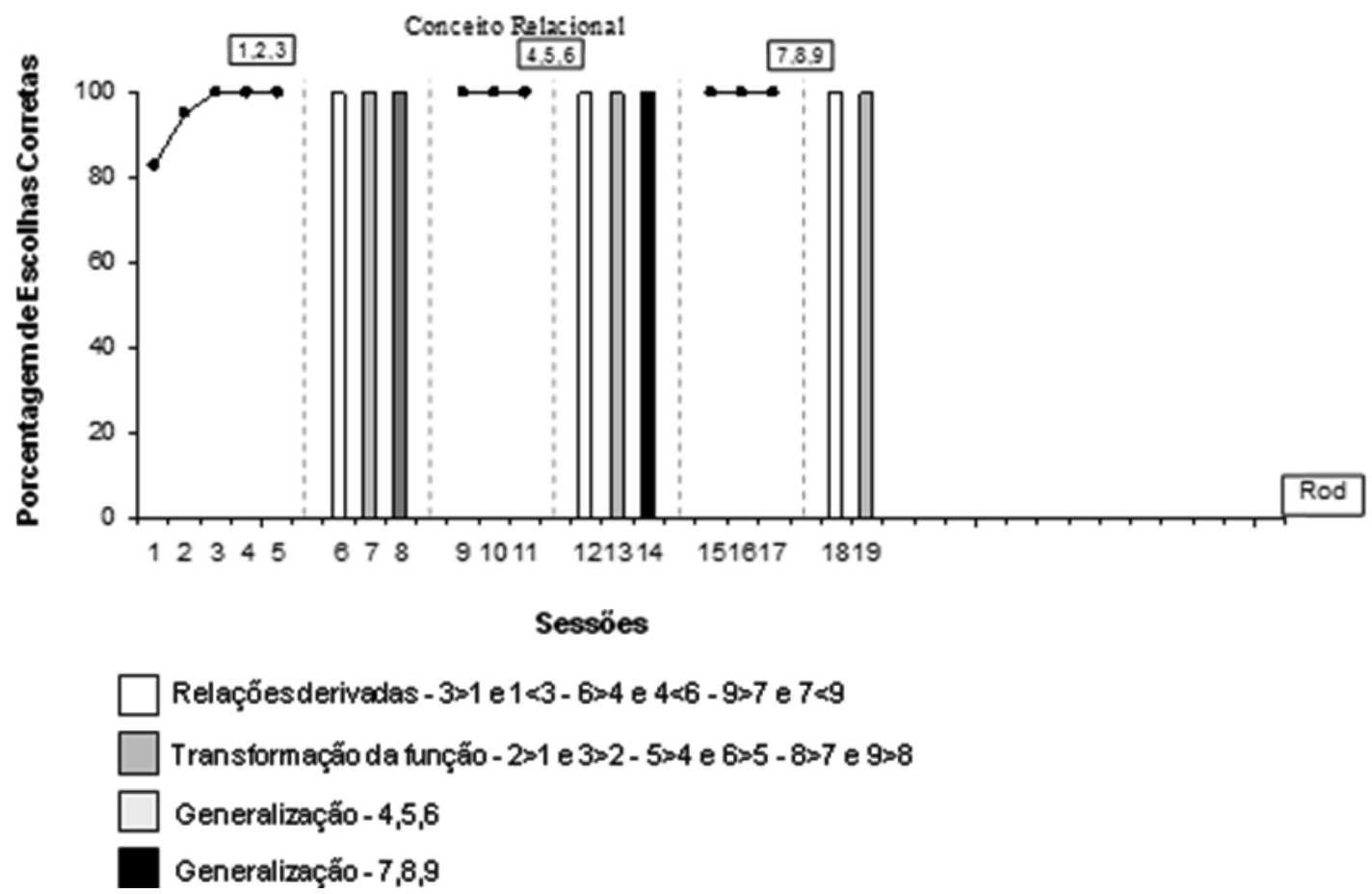

Figura 6. Desempenhos de Rod no ensino e testes das relações do conceito relacional para os valores um a nove. Os pontos ligados por linhas ilustram as relações ensinadas diretamente e as barras ilustram as relações testadas.

Na fase de ensino com os valores 1, 2, e 3, Jov e Jul adquiriram as relações $\mathrm{AB}$ em cinco sessões, as relações AC em duas sessões, e exibiram imediatamente as relações nos testes para verificar a emergência de relações de equivalência, de nomeação e contagem. Na fase de testes para verificar a emergência dos conceitos numéricos para os valores 4,5 , e 6 o desempenho de Jul foi $100 \%$ consistente. O de Jov, no entanto, foi consistente para as relações $\mathrm{AB}, \mathrm{AC}$ e $\mathrm{BD}$, mas apresentou-se ao nível do acaso para as relações $\mathrm{CD}, \mathrm{BC}$ e $\mathrm{CB}$ (ver painel superior das Figuras 4 e 5 , respectivamente).

$\mathrm{Na}$ fase de ensino das relações de conceito relacional, Jul e Jov adquiriram o critério em seis e cinco sessões, respectivamente, e os resultados nos testes de relações derivadas envolvendo as relações $/ 3>1 /$ e $/ 1<3 /$ mostraram-se dentro do critério para os dois participantes. Os testes de transformação da função envolvendo as relações $/ 2>1 /$ e $/ 3>2 /$ se apresentaram dentro do critério para Jul, mas não para Jov. Nas relações de generalização para os valores 4,5 , e 6 relativas a $/ 4<5 /$ e $/ 5<6 /$, os dois participantes mostraram desempenho abaixo do critério. Os resultados de generalização tanto de conceito numérico como de conceito relacional serviram como linha de base para o ensino desta relação na fase seguinte (painel intermediário).

Jul e Jov apresentaram as relações AB e AC para os valores 4, 5, e 6 após, respectivamente, quatro e oito sessões. No entanto, Jov recebeu um procedimento adicional antes que o critério pudesse ser observado. Este procedimento consistiu do desmembramento da relação $\mathrm{AB}$ em blocos. Inicialmente a sessão incluiu somente as tentativas das relações A4B4 e A5B5, em número idêntico de tentativas. Após o ensino dessas relações, foi introduzido o ensino das relações A5B5 e A6B6. Quando o participante atingiu o critério, passou-se para o ensino das relações A4B4 e A6B6 e, finalmente, das relações A4B4, A5B5 e A6B6, em tentativas randomizadas.

Os testes de equivalência para 4, 5, e 6 foram apresentados em seguida, tendo sido observado critério para todas as relações testadas. Os testes de generalização de conceito numérico para os valores 7, 8, e 9, apresentados na sequência (no painel intermediário) mostraram resultados dentro do critério para todas as relações, com exceção de BD, para Jul, e CD, para Jov. 
Os conceitos relacionais relativos aos valores 4,5 , e 6 , e às relações $/ 4<5 /$ e $/ 5<6 /$ foram adquiridos pelos dois participantes. $\mathrm{O}$ desempenho de Jul e Jov tanto nas relações derivadas $(/ 4<6 /$ e /6>4/), quanto nas relações de transformação de função $(/ 5>4 /$ e /6 $>5 /)$, foi dentro do critério. Nos testes de generalização para os valores 7,8 , e 9 , para as relações $/ 7<8 /$ e $/ 8<9 /$, o desempenho de ambos participantes foi abaixo do critério.

Os painéis inferiores das Figuras 4 e 5 mostram o desempenho de Jul e Jov para conceito numérico relativo aos valores 7,8 , e 9 . Jul e Jov apresentaram critério nas relações $\mathrm{AB}$ e $\mathrm{AC}$, e critério nas relações derivadas testadas $(\mathrm{CD}, \mathrm{BC}$ e CB), com exceção da relação DB para Jov. Nas relações envolvendo o conceito relacional, os dois participantes exibiram as relações ensinadas e apresentaram desempenho dentro do critério nos testes de relações derivadas $(/ 7<9 /$ e $/ 9>7 /)$ e de transformação de função $(/ 8>7 /$ e $/ 9>8 /)$.

O participante Rod, diferentemente dos demais, apresentou todas as relações testadas envolvendo os valores de 1 a 9 nos conceitos numéricos. A Figura 6 mostra os resultados de Rod para o ensino e testes dos conceitos relacionais envolvendo os mesmos valores. Após a demonstração de critério dos conceitos relacionais envolvendo os valores 1,2 , e 3, Rod apresentou desempenho dentro do critério nos testes de relações derivadas, transformação de função e generalização, o mesmo acontecendo com os valores 4,5 , e 6 , e também para os valores 7,8 , e 9 .

\section{Discussão}

Os três participantes apresentaram, ao final do estudo, quer por via do ensino direto, quer por transferência do controle das funções para novos estímulos, os conceitos numéricos envolvendo os valores de 1 a 9. Também adquiriram o conceito relacional /menor-que/ diretamente ensinado por meio de procedimentos baseados em múltiplos exemplares de tarefas modificadas de escolha de acordo com o modelo, para se adequar à teoria dos quadros relacionais. Esses resultados confirmam, mais uma vez, a robustez dos procedimentos baseados no paradigma de equivalência de estímulos para o ensino de conceitos numéricos para indivíduos com deficiência intelectual (Gast, Vanbiervliet, \& Spradlin, 1979; Green, 1992; Rossit, 2003) e, em combinação, de conceitos relacionais baseados parcialmente no paradigma de equivalência e, parcialmente baseados na teoria dos quadros relacionais. O presente estudo inovou quanto à aplicação conjunta e metodologicamente integrada da tecnologia derivada das pesquisas sobre equivalência de estímulos (Sidman, 1994) e da RFT (Roche \& Barnes-Holmes, 1996; Roche et al., 2000), ao ensino de conceitos matemáticos para indivíduos com deficiência intelectual.

Rod foi o único participante a apresentar no pré-teste as relações relativas ao conceito numérico envolvendo os valores de 1 a 9 . No entanto, não apresentou critério de desempenho nas relações de conceitos relacionais. $\mathrm{O}$ ensino dessas relações com os valores 1,2 , e 3 foi realizado rapidamente, e a transferência para os valores 4 , 5, e 6 e 7, 8, e 9 foi observada imediatamente, assim como, nos testes de generalização, de relações derivadas e de transformação de função. Em função desses resultados, é possível supor que os resultados do pré-teste tenham sido enviesados pela dificuldade com que o jovem com deficiência intelectual tenha apresentado no entendimento da tarefa. Tendo em vista o desempenho de Rod nos pré-testes e no ensino e testes dos conceitos relacionais, é de se supor que houve necessidade apenas de que este participante aprendesse a tarefa envolvida nas sessões de ensino e testes dos conceitos relacionais. Rod era o participante mais velho e, durante o estudo, estava cursando a $4^{\mathrm{a}}$ série de ensino regular.

A extensão com que houve transferência da função de equivalência dos valores 1,2 , e 3 para 4, 5, e 6 e desses para 7, 8, e 9 para Jul e, em menor extensão, para Jov é motivo de discussão. Os resultados de Jul e, sobretudo, os de Jov sugerem que o ensino direto das relações $\mathrm{AB}$ e $\mathrm{AC}$ foi fundamental para a emergência das relações de equivalência testadas e, também, para fazer evocar as relações completas de tato, envolvendo comportamento de falante e ouvinte, dentro do contexto de matching-to-sample e de testes. No entanto, ainda para Jov, foi somente a partir da emergência do tato en- 
volvendo os estímulos para os valores 4,5 , e 6 , que as relações de equivalência surgiram para esses valores, e também a emergência das relações envolvendo os valores 7, 8, e 9. Esses dados, no entanto, requerem replicação com participantes com repertório de entrada mais restrito que a dos participantes do presente estudo, para que esta relação possa ser sugerida com base em evidências mais sólidas.

A extensão com que houve transferência do responder relacional de /menor-que/ para / maior-que/ para relações não diretamente ensinadas é motivo de discussão.

No presente estudo, a fase inicial consistiu de treino da tarefa de escolha de acordo com o modelo utilizando como estímulos figuras familiares. Outros tipos de tarefas, inclusive algumas das utilizadas nas fases seguintes de ensino e testes, não foram diretamente ensinadas. Dentre essas, não foram ensinadas as tarefas envolvendo relações entre palavras ditadas-figuras, estímulos visuais (figuras e algarismos) e relação de tato, e estímulos visuais (figuras e algarismos) e contagem, assim como, tarefas envolvendo as relações de maior e menor utilizadas nas fases seguintes de ensino e de testes. Em estudos translacionais ou aplicações práticas derivadas, a exposição aos diferentes tipos de tarefa é fundamental.

A extensão em que houve transferência para novos conceitos relacionais é também alvo de discussão.

Para todos os participantes, o ensino da rede de relações compreendendo não só a formação do conceito de alguns números, através da formação de classe de equivalência de estímulos, mas também um repertório verbal de tato, de nomeação e de contagem foi considerado importante, condição minimamente necessária, para prosseguir no ensino de conceitos relacionais envolvendo os mesmos números. No entanto, não foi possível avaliar, para Rod, a importância dessas relações, por ele já apresentar no início do estudo a maior parte do repertório a ser ensinado.

Jul e Jov, por outro lado, novamente apresentaram algumas características comuns de resultados e por isso serão discutidos conjuntamente. Primeiramente serão considerados os re- sultados de aquisição e emergência de conceitos numéricos.

Por não terem apresentado, no pré-teste, o repertório relativo ao conceito numérico a partir dos valores 1, 2, e 3, Jul e Jov foram submetidos ao ensino direto das relações $\mathrm{AB}$ e $\mathrm{AC}$, após o qual foram testadas as relações $\mathrm{BC}, \mathrm{CB}, \mathrm{BD}$ e $\mathrm{CD}$, tendo os dois participantes exibido as relações de equivalência e adquirido o conceito numérico para esses valores.

$\mathrm{O}$ repertório de conceito numérico envolvendo valores 4,5 , e 6 e 7, 8, e 9 também emergiu nos testes para ambos participantes, em menor extensão nas relações com os valores 4 , 5, e 6 para Jov. Especificamente, Jov não exibiu as relações de nomeação do algarismo ou de equivalência inicialmente para 4,5 , e 6 . Ainda que as possíveis explicações para esse fato fiquem apenas em nível de especulação, o valor potencial prático educacional que o procedimento apresenta em termos de emergência de novos comportamentos e economia de ensino é bastante significativo. Jul apresentou essa mesma característica quando as relações com os valores 7,8 , e 9 foram introduzidas como procedimento de ensino.

Os resultados que nos parecem mais interessantes ainda são aqueles relacionados a relações derivadas, transformação de função e generalização. Para Jul e Jov, após o ensino das relações $/ 1<2 /$ e $/ 2<3 /$ foi observada a emergência das relações transitivas derivadas $/ 1<3 /$ e $/ 3>1 /$ e também a emergência das relações com função transformada $/ 2>1 /, / 3>2 /$. Adicionalmente, foi observada a generalização do responder relacional envolvendo os valores 4, 5, e 6 e 7, 8, e 9 no desempenho de Jul, mas em menor extensão para Jov.

A emergência das relações envolvendo os valores 4,5 , e 6 para Jul, poderia ser explicada pela presença do critério para o conceito de número desde o teste de generalização para estes valores (painel superior Figura 4). O resultado aponta que a aquisição do conceito de número é essencial para a aquisição de conceitos mais complexos, como é o caso do conceito relacional de /menor-que/ e /maior-que/, o que implica a necessidade dos estímulos serem analisados in- 
dependentemente, comparados entre si e selecionados de acordo com a dica contextual.

Os conceitos relacionais /maior-que/ e / menor- que/ pressupõem uma compreensão do conceito numérico para poderem, também, explicitar a dimensão de grandeza e magnitude de um valor em relação ao outro.

A presente pesquisa pode ainda contribuir para aumentar a credibilidade na aprendizagem de alunos com deficiência intelectual e em procedimentos que levam a tal aprendizagem. Os procedimentos fundamentados nos princípios da análise do comportamento permitiram que a aprendizagem ocorresse e que as novas aquisições fossem mantidas ao longo do tempo no repertório do indivíduo.

Utilizou-se uma mescla entre o paradigma de equivalência de estímulos e a Teoria dos Quadros Relacionais, entretanto, os termos usados para descrever as propriedades de uma relação equivalente - reflexividade, simetria e transitividade - não se aplicam para o tipo de desempenho relacional demonstrado no presente experimento. Whelan et al. (2006) discutem que as propriedades de responder para /menor-que/ e / maior-que/ têm mais em comum com relações temporal e de ordem. Segundo esses autores, essas relações são arreflexivas, assimétricas, transitivas e conectadas.

Whelan et al. (2006) justificam suas colocações identificando algumas razões: (a) referem que a relação que o estímulo A tem com ele mesmo não é reflexiva, pois algo não pode ser / maior-que/ ou /menor-que/ ele mesmo; (b) se A é /maior-que/ $\mathrm{B}$, isto na significa que possamos dizer que B é /maior-que/ A, portanto, as relações não são simétricas; (c) as relações são transitivas, se B é/maior-que/ A e C é/maior-que/ B, e então $\mathrm{C}$ é /maior-que/ $\mathrm{A}$; (d) as relações também são conectadas ${ }^{5}$.

Os dados presentes, entretanto, suportam e estendem não somente estudos dentro da literatura da teoria dos quadros relacionais (Whelan et

\footnotetext{
5 "Todo estímulo que participa em uma relação específica /maior que/ ou /menor que/ é relacionado com cada um dos outros, e a relação é portanto, conectada" (Dymond \& Barnes, 1995, p. 183).
}

al., 2006), mas também aqueles estudos que têm se preocupado em estender o conjunto de análises da teoria das classes de equivalência de Sidman para relações não equivalentes e, sugerem ainda, que os estímulos podem se recombinar de forma ainda não totalmente esclarecida.

Diversos estudos (Barnes-Holmes, Barnes-Holmes, \& Cullinan, 2001; Dymond \& Barnes-Holmes, 1995, 1996; Roche \& Barnes-Holmes, 1996; Roche et al., 2000) de estímulos relacionais derivados foram conduzidos sob a influência da Teoria dos Quadros Relacionais. Essa teoria explicitamente reconhece e se detém a explicar a emergência de uma variedade de relações de estímulos derivadas além da equivalência.

Mas como explicar que um par de estímulos que não tenha sido relacionado explicitamente durante uma situação de treino possa se mostrar presente. Tem-se como exemplo, o treino das relações $/ 1<2 / \mathrm{e} / 2<3 /$. Como explicar a transformação da função com a derivação de respostas do tipo $/ 2>1 /$ e $/ 3>2 /$ ?

Os resultados do estudo são consistentes com os achados de Dougher, Hamilton, Fink, e Harrington (2007) e Whelan et al. (2006).

Dougher et al. (2007) estabeleceu uma rede relacional de três elementos (estímulos pequeno, médio e grande) usando procedimentos semelhantes aos descritos no presente estudo (ou seja, testes e treinos relacional arbitrário e não-arbitrário) e demonstrou que as funções de estímulo operante podem ser transformadas de acordo com relações não-equivalentes, ou o que os autores se referem como "estímulo relacional generalizado". Em uma tarefa padrão, o estímulo modelo "médio" foi utilizado para treinar a pressão na barra até uma determinada taxa. No teste para a transformação da função, os participantes pressionaram mais lentamente na presença do estímulo modelo "pequeno" e pressionaram mais rapidamente na presença do estímulo modelo "grande", independente do fato deles não terem emitido previamente a resposta de pressionar a barra na presença destes estímulos. Os dados de Whelan et al. (2006) apoiam e estendem estes achados por: (a) demonstrar a transformação de funções consequentes, em vez de funções discriminativas, (b) aumentar o número de estímulos 
na rede relacional de três para sete, e (c) mostrar generalização do desempenho derivado de um contexto de teste relacional arbitrário para uma tarefa baseada no esquema de operante livre.

Os dados do presente estudo mostram que alterar as contingências da linha de base para relações com estímulos múltiplos produz mudanças confiáveis e previsíveis para as respostas relacionais no quadro de comparação, de entendimento mútuo, derivação combinatória e a transformação da função.

A Teoria dos Quadros Relacionais mostra que variáveis adicionais podem interagir para a emergência de novos tipos de relações derivadas (Ninness, et al., 2005; Pelaez \& O'Hora, 2004). Para esses autores, a combinação é um processo de formar uma estrutura de conhecimento nova e integrada a partir de uma informação codificada, entendida como 'equivalente a' ou 'vínculo combinatorial', então tem-se que, se $\mathrm{A}<\mathrm{B}$, e $B<C$, então $A<C$, substituindo as letras por valores encontra-se $1<2$; e $2<3$, então, $1<3$. Um outro processo envolve a comparação da nova estrutura de conhecimento com a informação codificada previamente, se $\mathrm{A}=\mathrm{D}$ (tem-se que $1=1$ ), então $\mathrm{D}<\mathrm{B}(1<2)$.

Além da evidência do responder relacional derivado, o presente estudo constatou também a manutenção das relações condicionais, as quais foram sistematicamente avaliadas uma semana após o treino, com a reapresentação da última relação adquirida na sessão anterior. Constatou-se que Jul, Jov e Rod sempre mantiveram o critério.

$\mathrm{O}$ aspecto da manutenção de repertórios previamente adquiridos, não tem recebido a atenção necessária por parte dos pesquisadores que, frequentemente, não avaliam a retenção ao longo do tempo. Esta é uma característica importante quando se tem como participantes pessoas que culturalmente são reconhecidas por suas limitações cognitivas e dificuldades na aquisição e manutenção de habilidades complexas. Os dados do presente estudo indicam, também, que os procedimentos utilizados foram eficientes para a aquisição e manutenção de relações complexas, como é o caso dos conceitos relacionais.

Um comportamento, evidenciado em Jul, Jov e Rod durante a realização das tarefas, foi verbalizar em voz alta os passos que realizavam em cada tentativa: nomear em voz alta o algarismo ou as quantidades, repetir a dica contextual emitida pelo computador (/menor-que/ ou / maior-que/), realizar a operação em voz alta (ex: lum é menor que... dois/), verbalizar o valor do estímulo que considera correto e tocá-lo como escolha. Na presença desses comportamentos, foi observada maior ocorrência de escolhas corretas, entretanto, novas investigações serão necessárias para poder explicar esses aspectos.

A resposta verbal discriminada, por exemplo, vocalizar/dois/, na presença de quantidades ou algarismo "2" é considerado como o tatear o 'dois'. Um comportamento ecóico ocorria imediatamente após a instrução do computador / menor-que/ e, em seguida, o tato recém-adquirido pode ser incorporado em um mando novo, apontando e tocando o estímulo que considerava correto.

Os dados encontrados no presente estudo reiteram os achados por Barnes-Holmes et al. (2001). Os autores descrevem que "pessoas com um vocabulário altamente elaborado tenderão a ter um alto repertório de responder relacional". O'Hora, Pelaez, e Barnes-Holmes (2005) referem que o desempenho em tarefas de responder relacional derivado varia de acordo com as performances de linguagem. Os dados mostraram que a aquisição de conceitos relacionais estava diretamente relacionado ao desempenho em sub-testes verbais. Participantes que falharam nestes teste tiveram maiores dificuldades na aquisição dos conceitos relacionais. Há evidências, portanto, que o responder relacional derivado e o desempenho em tarefas complexas de linguagem estão fortemente relacionados.

\section{Considerações Gerais}

O presente estudo se propôs a analisar o repertório matemático dos conceitos de número e relacional /menor-que/; verificar a emergência das relações derivadas, a transformação da função para /maior-que/ e a generalização para outros valores.

A tecnologia utilizada para a avaliação e o ensino direto de algumas relações possibilitou a 
evidência de respostas derivadas e a transformação da função.

O uso do computador, do programa computacional Mestre ${ }^{\circledR}$, do ensino informatizado, da estrutura das tarefas mostraram-se viáveis e eficientes para avaliar e ensinar, permitindo, também, que os participantes ficassem sob o controle quase estrito do conteúdo da tarefa, pois verificou-se a emergência, derivação de respostas, transformação de função e manutenção das relações que foram avaliadas imediatamente após o término do ensino direto, e uma semana após a aquisição inicial.

\section{Referências}

Barnes-Holmes, Y., Barnes-Holmes, D., \& Cullinan, V. (2001). Education. In S. C. Hayes, D. Barnes-Holmes, \& B. T. Roche (Eds.), Relational frame theory: A post-Skinnerian account of human language and cognition (pp. 181-196). New York: Kluwer Academic.

De León, N. P. A. (1998). Aquisição de habilidades básicas de matemática através da formação de equivalência em crianças pré-escolares (Dissertação de mestrado não-publicada, Universidade Federal de São Carlos, SP, Brasil).

Dougher, M. J., Hamilton, D. A., Fink, B., \& Harrington, J. (2007). Transformation of the discriminative and eliciting functions of generalized relational stimuli. Journal of the Experimental Analysis of behavior, 2, 179-198. doi:10.1901/ jeab.2007.45-05

Dymond, S., \& Barnes-Holmes, D. (1995). A transformation of self-discrimination functions in accordance with the derived stimulus relations of Sameness, More than, and Less than. Journal of the Experimental Analysis of Behavior, 64, 163 184. doi:10.1901/jeab.1995.64-163

Dymond, S., \& Barnes-Holmes, D. (1996). A transformation of self-discrimination response functions in accordance with the arbitrarily applicable relations of sameness and opposition. The Psychological Record, 46, 271-300.

Escobal, G., \& Goyos, A. C. N. (2008). Análise das variáveis determinantes do comportamento de escolha entre alternativas de trabalho em adultos com deficiência mental. Revista Brasileira de Análise do Comportamento, 4(1), 71-87.
Gast, D. L., Vanbiervliet, A., \& Spradlin, J. E. (1979). Teaching number-word equivalences: A study of transfer. American Journal of Mental Deficiency, 83, 524-527.

Goyos, C., \& Almeida, J. C. B. (1994). Mestre (Versão 1.0). [Computer software]. São Carlos, SP: Mestre Software. Recuperado em www.geocities.com/EnchantedForest/Glade/1252

Green, G. (1992). Stimulus control technology for teaching number/quantity equivalences. In Proceedings of the Conference os the National Association for Autism (pp. 51-64). Melboume, Australia: Victorian Autistic Children's, \& Adults'.

Green, G., Stromer, R., \& Mackay, H. (1993). Relational learning in stimulus sequences. The Psychological Record, 43, 599-616.

Hayes, S. C., Barnes-Holmes, D., \& Roche, B. T. (2001). Relational Frame Theory: A post-Skinnerian account of human language and cognition. New York: Plenum.

Horne, P. J., \& Lowe, C. F. (1996). On the origins of naming and other symbolic behavior. Journal of the Experimental Analysis of Behavior, 65, 185241. doi:10.1901/jeab.1996.65-185

Maydak, M., Stromer, R., Mackay, H., \& Stoddard, L. (1995). Stimulus classes in matching to sample and sequence production: The emergence of numeric relations. Research in Developmental Disabilities, 16, 179-204. doi:10.1016/08914222(95)00008-B

Neef, A. N., Nelles, D. E., Iwata, B. A., \& Pace, T. J. (2003). Analysis of precurrent skills in solving mathematics story problems. Journal of Applied Behavior Analysis, 36, 21-33. doi:10.1901/ jaba.2003.36-21

Ninness, C., Rumph, R., McCuller, G., Vasques, E., Harrison, C., Ford, A. M., Capt, A., ...Bradfield, A. (2005). A relational frame and artificial neural network approach to computer-interactive mathematics. The Psychological Record, 55, 135-153.

O’Hora, D., Pelaez, M., \& Barnes-Holmes, D. (2005). Derived relational responding and performance on verbal subtests of the WAIS-III. The Psychological Record, 55, 155-175.

O’Hora, D., Roche, B., Barnes-Holmes, D., \& Smeets, P. M. (2002). Response latencies to multiple derived stimulus relations: Testing two predictions of relational frame theory. The Psychological Record, 52, 51-75. 
Pelaez, M., \& O’Hora, D. (2004, August). Relational frames and human intelligence. Paper presented at the XIII Encontro da Associação Brasileira de Psicoterapia e Medicina Comportamental \& II International Congress of Association for Behavior Analysis. Campinas, SP: Associação Brasileira de Psicologia e Medicina Comportamental.

Pereira, J. A., \& Winton, A. S. (1991). Teaching and remediation of mathematics: A review of behavioral research. Journal of Behavioral Education, 1, 5-36. doi:10.1007/BF00956752

Prado, P. S. T., \& de Rose, J. C. (1999). Conceito de número: Uma contribuição da Análise Comportamental da Cognição. Psicologia: Teoria e Pesquisa, 15(3), 227-235. doi:10.1590/S010237721999000300006

Reilly, T., Whelan, R., \& Barnes-Holmes, D. (2005). The effect of training structure on the latency of responses to a five-term linear chain. The Psychological Record, 55, 233-249.

Ribeiro, M. P. L., Assis, G., \& Enumo, S. R. F. (2007). Comportamento matemático: Relações ordinais e inferência transitiva em pré-escolares. Psicologia: Teoria e Pesquisa, 23(1), 25-32. doi:10.1590/S0102-37722007000100004

Roche, B., \& Barnes-Holmes, D. (1996). Arbitrarily applicable relational responding and sexual categorization: A critical test of the derived difference relation. The Psychological Record, 46, 489-507.

Roche, B., Barnes-Holmes, D., Smeets, R. M., Barnes-Holmes, Y., \& McGeady, S. (2000). Contextual control over the derived transformation of discriminative and sexual arousal functions. The Psychological Record, 50, 267-291.

Rossit, R. A. S. (2003). Matemática para deficientes mentais: Contribuições do paradigma de equivalência de estímulos para o desenvolvimento $e$ avaliação de um currículo (Tese de doutorado, Universidade Federal de São Carlos, SP, Brasil). Recuperada em http://www.bv.fapesp.br/pt/ publicacao/5135/matematica-para-deficientes-mentais-contribuicoes-do-paradig/

Rossit, R. A. S., \& Goyos, C. (2009). Deficiência intelectual e aquisição matemática: Currículo como rede de relações condicionais. Psicologia Escolar e Educacional, 13(2), 213-225. doi:10.1590/ S1413-85572009000200003

Sidman, M. (1971). Reading and auditory-visual equivalences. Journal of Speech and Hearing Research, 14, 5-13. doi:10.1044/jshr.1401.05
Sidman, M. (1994). Equivalence relations and behavior: A research story. Boston, MA: Authors Cooperative.

Steele, D., \& Hayes, S. C. (1991). Stimulus equivalence and arbitrarily applicable relational responding. Journal of the Experimental Analysis of Behavior, 56, 519-555. doi:10.1901/ jeab.1991.56-519

Stoddard, L. T., Bradley, D. P., \& McIlvane, W. J. (1987). Stimulus control of emergent performances: Teaching money skills. In J. A. Mulick \& R. F. Antonak (Eds.), Transitions in mental retardation: Vol. 2. Issues in therapeutic intervention (pp. 113-149). Norwood, NJ: Ablex.

Stoddard, L. T., Brown, J., Hurlbert, B., Manoli, C., \& McIlvane, W. J. (1989). Teaching money skills through stimulus class formation, exclusion and component matching methods: Three case studies. Research in Developmental Disabilities, 10(4), 413-439. doi:10.1016/08914222(89)90041-3

Stromer, R., Mackay, H. A., \& Stoddard, L. T. (1992). Classroom applications of stimulus equivalence technology. Journal of Behavioral Education, 2, 225-256. doi:10.1007/BF00948817

Wechsler, D. (1991). Wechsler Intelligence Scale for Children - Third edition. San Antonio, TX: The Psychological Corporation.

Whelan, R., \& Barnes-Holmes, D. (2004). The transformation of consequential functions in accordance with the relational frames of same and opposite. Journal of the Experimental Analysis of Behavior, 82(2), 177-195. doi:10.1901/ jeab.2004.82-177

Whelan, R., Barnes-Holmes, D., \& Dymond, S. (2006). The transformation of consequential function in accordance with the relational frame of more-than and less-than. Journal of the Experimental Analysis of Behavior, 86, 317-335. doi:10.1901/jeab.2006.113-04 